\title{
Correlation of Mitral Annular Plane Systolic Excursion (MAPSE) and Tissue Doppler peak Systolic Velocity with Left Ventricular Systolic Function
}

\begin{abstract}
Background: Estimation of left ventricular function has major diagnostic and prognostic importance in cardiac patients. The most commonly used measure of the systolic function of the left ventricle is ejection fraction (EF), however, the accuracy is limited especially in patients with poor image quality. Mitral annular plane systolic excursion (MAPSE) and tissue Doppler peak systolic velocity (TD PSV) are measurements of longitudinal function which has been shown to correlate with LV global systolic function.
\end{abstract}

Objective: To validate the correlation of mitral annular plane systolic excursion (MAPSE) and TD PSV (Sm) at the medial and lateral mitral annuli as surrogates for estimation of LV systolic function.

Methods: The study included 200 patients divided into 2 groups; Group (A) which included 100 patients with normal EF as assessed by biplane Simpson's method (defined as EF $>50 \%$ ) and Group (B) which included 100 patients with reduced ejection fraction (defined as $\mathrm{EF}<50 \%$ ). MAPSE and TD PSV (Sm) were recorded at medial and lateral mitral annuli in the apical four-chamber approach.

Results: The cut off value of MAPSE at medial annulus of $\leq 7 \mathrm{~mm}$ suggested an impaired systolic function with sensitivity of $73 \%$ and specificity of $100 \%$. While the cut off value of MAPSE at lateral annulus of $\leq 10 \mathrm{~mm}$ suggested an impaired systolic functions with sensitivity of $82 \%$ and specificity of 93\%. TD PSV (Sm) cut off value at the Medial and lateral annuli $\leq 7 \mathrm{~cm} / \mathrm{s}$ suggested an impaired LV systolic function with sensitivity of $91 \%$ and specificity of $85 \%$ for the medial annulus and with sensitivity of $72 \%$ and specificity of $93 \%$ for the lateral annulus. When the cut-off values of both parameters (TD PSV and MAPSE) were combined to detect $\mathrm{LVEF}<50$, the sensitivity and specificity increased to $95.9 \%$ and $100 \%$ respectively for the Medial Mitral annulus and $85.5 \%$ and $97.7 \%$ respectively for the lateral Mitral annulus

Conclusion: Combining the measurement of both MAPSE and Mitral annular TD PSV increased their sensitivity and specificity for predicting normal or subnormal EF.

Keywords: MAPSE; PSV; Tissue Doppler; left ventricle function; Ejection fraction

Research Article
Volume 10 Issue 1 - 2017
Hazem Khorshid ${ }^{1 *}$, Bassem Wadeea ${ }^{1}$ and
Enas Sabry ${ }^{2}$
${ }^{1}$ Department of Cardiology, Ain Shams University Hospital,
Egypt
${ }^{2}$ Police Hospital, Cardiology Department, Egypt
*Corresponding author: Hazem M Khorshid, Department of
Cardiology, Ain Shams University Hospital, Cairo, Egypt, Tel:
202 24821894, Email: hazemkhorshid@yahoo.com
Received: October 22, 2017 | Published: November 06,
2017

Abbreviations: EF: Ejection Fraction; MAPSE: Mitral Annular Peak Systolic Excursion; TD: Tissue Doppler; PSV: Peak Systolic Velocity; LV: Left Ventricular

\section{Introduction}

The assessment of left ventricular systolic function is the most common indication for performing an echocardiogram, however, no perfect measure of systolic function exists and there are limitations to currently available methods [1]. The most widely used measurement of left ventricular systolic function is the ejection fraction, which also provides important prognostic information [2]. However, its accuracy is limited especially in patients with poor image quality when the endocardial border is not clear enough for tracing where the prevalence of poor image quality was $23-31 \%$ in most studies [3]. Moreover major interand intra-observer variability exists [4].

Mitral annular plane systolic excursion (MAPSE) is an easy measure to assess longitudinal function and has been shown to correlate with LV function measurements and is very sensitive in different cardiac diseases [5,6]. Tissue Doppler imaging (TDI) also provides an easy method to measure peak systolic velocity of mitral annular longitudinal movement $\left(S^{\prime}\right)$, which is an excellent non-invasive index of global LV systolic function [7]. Combining both indices (MAPSE and Mitral annular TD PSV) and correlating them to the EF was not reported before to the best of our knowledge. Hence the aim of the present study was to validate the correlation of mitral annular plane systolic excursion (MAPSE) by m-mode and Mitral annular TD PSV as predictors for EF. 


\section{Materials and Methods}

The present study included 200 patients with age ranging between 17-81years. The study was approved by the Ethics committee of Ain Shams University. The objectives of the project and study design were explained completely to the participants and informed consent was obtained from them as well as they were ensured their data would remain private. The patients were divided into 2 groups:

Group (A) which included 100 patients with normal ejection fraction (defined as $\mathrm{EF}>50 \%$ ) as assessed by biplane Simpson's method.

Group (B) which included 100 patients with sub normal ejection fraction (defined as $\mathrm{EF}<50 \%$ ) as assessed by biplane Simpson's method.

The patients were subjected to detailed history talking and thorough clinical examination. All patients underwent full twodimensional echocardiographic examination using GE vivid 7 machine, and measurements were obtained in accordance with the current American Society of Echocardiography guidelines [8]. Echocardiography was performed with the patients in the left lateral decubitus. Two-dimensional imaging examination was performed in the standard fashion in parasternal long-axis view and apical 4-chamber view. Optimization was performed using harmonic imaging, gain, dynamic range, frequency, sector width and focus to improve signal-to-noise ratio and provide optimal endocardial definition.

Echocardiograms were subject to careful visual analysis to detect regional contractile abnormalities which were excluded. LV systolic and diastolic volumes and ejection fraction were derived from apical 2 and 4-chamber views using the modified Simpson's rule algorithm. All echocardiographic and TD recordings were obtained during normal respiration.

\section{MAPSE (mitral annular peak systolic excursion)}

Displacement of the mitral annulus was measured in millimeters $(\mathrm{mm})$ by M-mode at two different points (medial and lateral mitral annuli) in the apical four-chamber view. The M-mode alignment was adjusted parallel to the LV walls along the ultrasonic beam. The systolic excursion of the mitral ring was measured from the lowest point of the terminal diastole to the highest point during systole (end of the T-wave in the ECG)

\section{TD Examination of mitral annulus}

TD of the mitral annulus was obtained from the apical 4-chamber view after filters were set to exclude high-frequency signals. A 5-mm sample volume was placed sequentially at the medial and lateral mitral annuli. PSV (Sm) measured in $\mathrm{cm} / \mathrm{sec}$ was recorded for 3 consecutive cardiac cycles at a sweep speed of $100 \mathrm{~mm} / \mathrm{s}$. The following patients were excluded from the study: patients with significant arrhythmia, congenital Heart disease, valvular stenosis, significant valvular regurgitation, prosthetic valves, regional wall motion abnormality and significant Mitral annular calcification. Also patients with poor echogenicity who were difficult to define the endocardial border were excluded.

\section{Statistical analysis}

Mann-Whitney and chi-square tests were used to assess the baseline variables (age, gender, Risk factors). Receiver operating characteristic (ROC) curves and Interactive dot diagrams were plotted to examine the ability of MAPSE and PSV (Sm) to differentiate patients with impaired systolic functions $(\mathrm{EF}<50 \%)$ from those with normal systolic function, and sensitivity and specificity were determined accordingly. All analyses were done using MedCalc v14.8.1 by MedCalc Software.

\section{Results}

There was no statistically significant difference between the 2 groups regarding age, gender and prevalence of HTN and dyslipidemia $(p>0.05)$. However prevalence of DM was statistically significantly higher in Group B as compared to group $A(p=0.002)$ (Table 1$)$. There was a statistically highly significant difference between the 2 studied groups regarding the measured echo parameters (EF\% measured by Simpson's method, TD PSV at medial and lateral Mitral annuli and MAPSE at medial and lateral Mitral annuli), being statistically significantly lower in group B as compared to Group A ( $\mathrm{p}<0.001)$ (Table 2).

Table 1: Demographic data and prevalence of Risk factors among the 2 studied groups.

\begin{tabular}{|c|c|c|c|c|}
\hline \multicolumn{2}{|c|}{} & Group A & Group B & p-value \\
\hline \multicolumn{2}{|c|}{ Age (years) } & $54.36 \pm 16.3$ & $59.32 \pm 9.2$ & 0.104 \\
\hline \multirow{2}{*}{ Gender } & Males & $38(38 \%)$ & $52(52 \%)$ & 0.064 \\
\cline { 2 - 5 } & Females & $62(62 \%)$ & $48(48 \%)$ & \\
\hline \multicolumn{2}{|c|}{ DM } & $20(20 \%)$ & $41(41 \%)$ & 0.002 \\
\hline \multicolumn{2}{|c|}{ HTN } & $41(41 \%)$ & $39(39 \%)$ & 0.885 \\
\hline \multicolumn{2}{|c|}{ Dyslipidemia } & $35(35 \%)$ & $40(40 \%)$ & 0.559 \\
\hline
\end{tabular}

DM: Diabetes Mellitus; HTN: Hypertension.

The ROC curve and Interactive dot diagram of MAPSE at the medial mitral annulus showed that the optimal cut off value for detecting subnormal LVEF ( $\mathrm{LVEF}<50 \%$ ) was $\leq 7 \mathrm{~mm}$ with sensitivity of $73 \%$ and specificity of $100 \%$ (Figures $1 \& 2$ ), (Table 3 ). The ROC curve and Interactive dot diagram of MAPSE at the lateral Mitral annulus showed that the optimal cut off value for detecting subnormal $\mathrm{EF}$ ( $\mathrm{LVEF}<50 \%$ ) was $\leq 10 \mathrm{~mm}$ with sensitivity of $82 \%$ and specificity of $93 \%$ (Figures $3 \& 4$ ), (Table $3)$. The ROC curve and Interactive dot diagram of TD PSV (Sm) at the medial and lateral Mitral annuli showed that the optimal cut off value for detecting subnormal EF (LVEF $<50 \%$ ) was $\leq 7 \mathrm{~cm} /$ sec with sensitivity of $91 \%$ and specificity of $85 \%$ for the medial annulus (Figures $5 \& 6$ ), (Table 3), and with sensitivity of $72 \%$ and specificity of $93 \%$ for the lateral mitral annulus (Figures 7 \& 8), (Table 3). When the cut-off values of both parameters (Mitral annular TD PSV and MAPSE) were combined for detecting subnormal (LVEF $<50 \%$ ) , the sensitivity and specificity increased to $95.9 \%$ and $100 \%$ respectively for the medial Mitral annulus and $85.5 \%$ and $97.7 \%$ respectively for the lateral Mitral annulus (Tables 4 \& 5). 


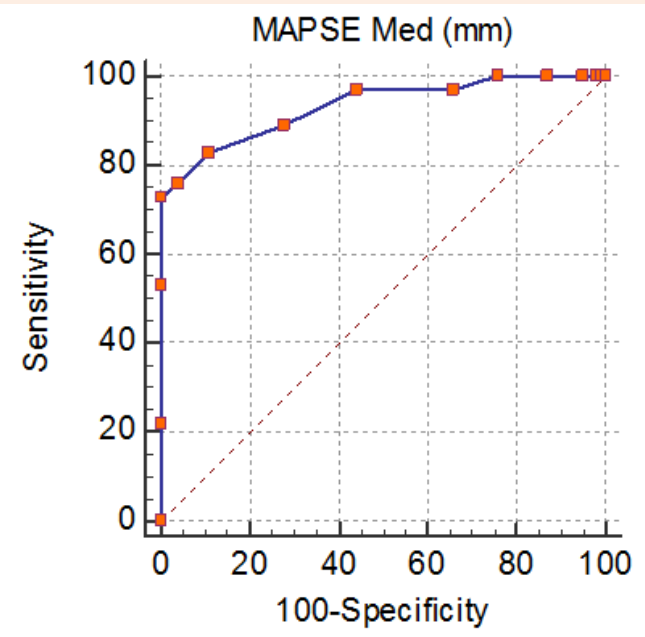

Figure 1: The ROC curve for the optimal cut-off point of MAPSE at the medial Mitral annulus.

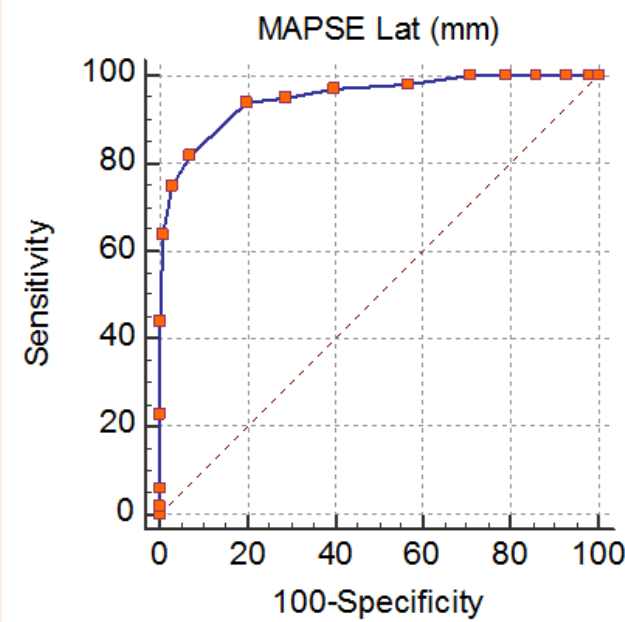

Figure 3: The ROC curve for the optimal cut-off point of MAPSE at the Lateral Mitral annulus.

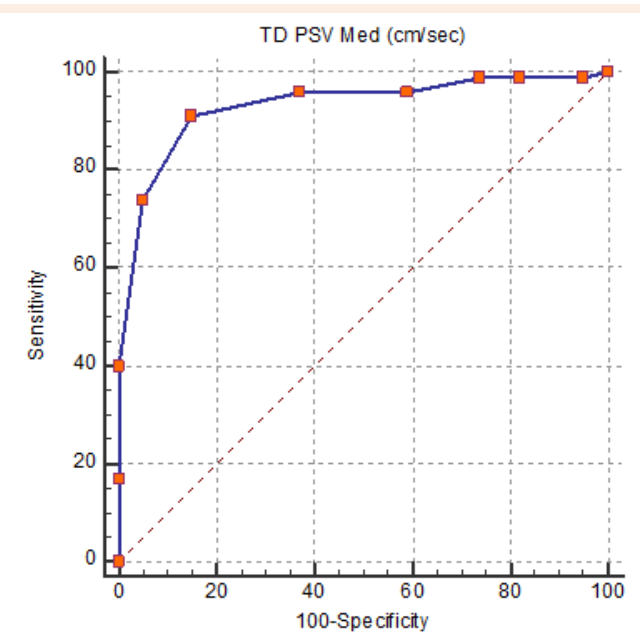

Figure 5: The ROC curve for the optimal cut-off point of TD PSV (Sm) at the Medial mitral annulus.

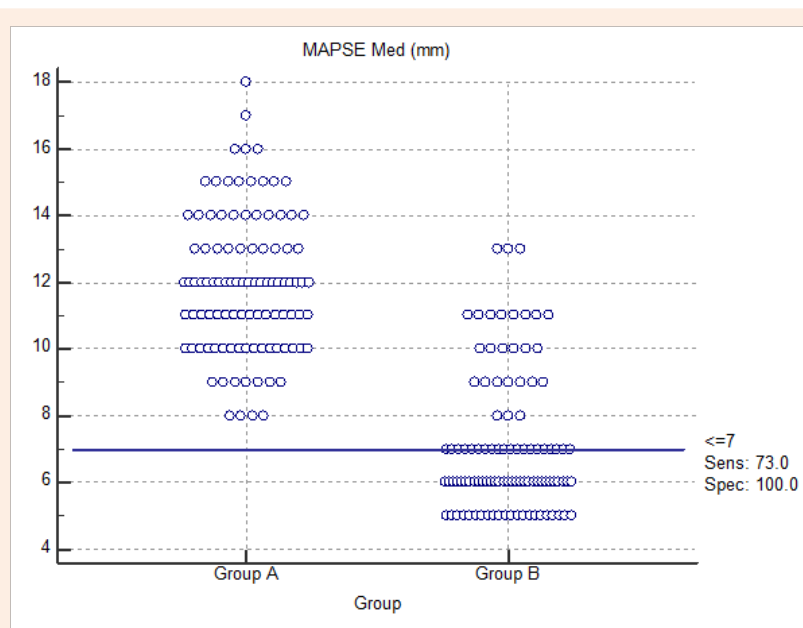

Figure 2: Interactive dot diagram for the cut-off point between normal and abnormal EF as regard to MAPSE at the Medial Mitral annulus.

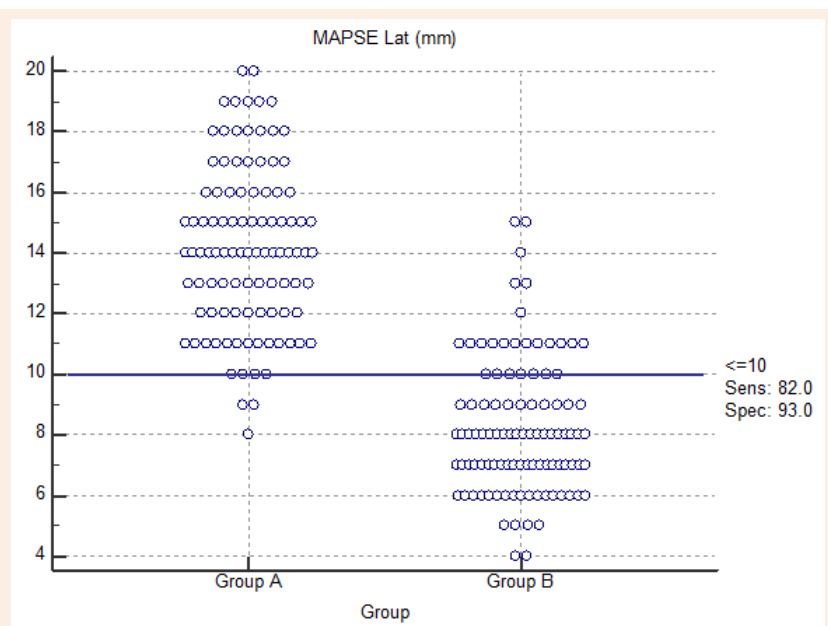

Figure 4: Interactive dot diagram for the cut-off point between normal and abnormal EF as regard to MAPSE at the Lateral Mitral annulus.

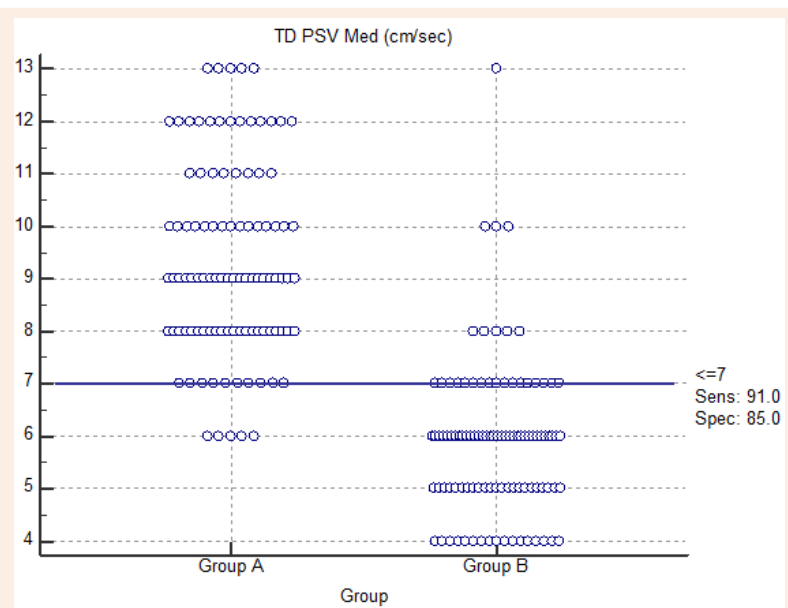

Figure 6: Interactive dot diagram for the cut-off point between normal and abnormal EF as regard to TD PSV (Sm) at the Medial Mitral annulus. 


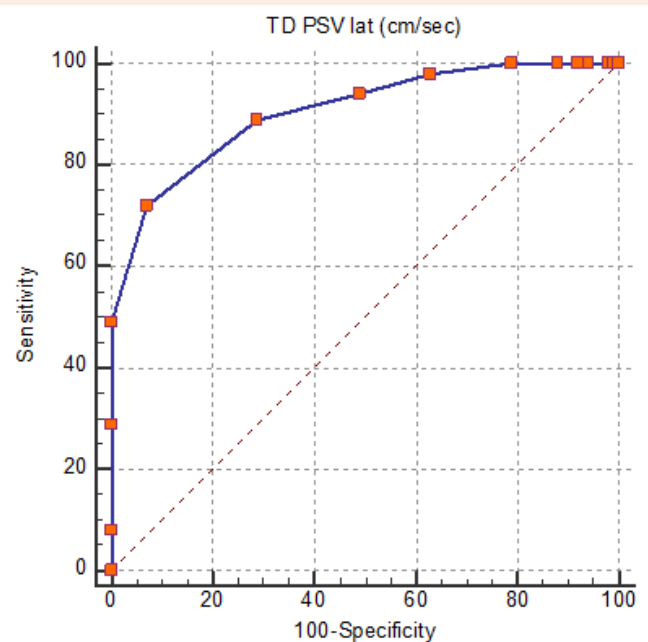

Figure 7: The ROC curve for the optimal cut-off point of TD PSV (Sm) at the Lateral Mitral annulus.

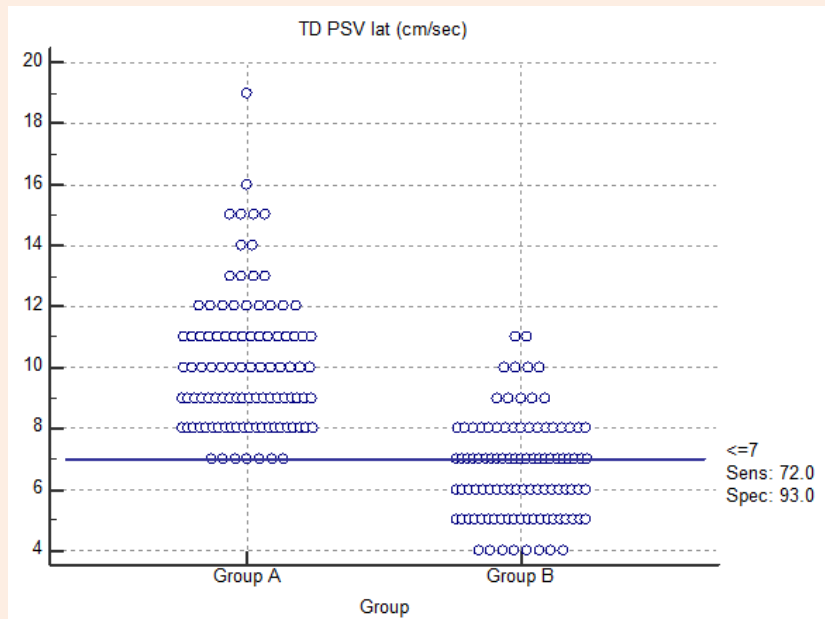

Figure 8: Interactive dot diagram for the cutoff point between normal and abnormal EF as regard to TD PSV (Sm) at the Lateral Mitral annulus.

Table 2: The Echocardiographic parameters of both studied groups.

\begin{tabular}{|c|c|c|c|c|c|c|}
\hline \multirow{2}{*}{ Parameters } & \multicolumn{2}{|c|}{ Group A } & \multicolumn{2}{c|}{ Group B } & \multicolumn{2}{c|}{ Independent t-test } \\
\cline { 2 - 7 } & Mean & SD & Mean & SD & t & p-value \\
\hline EF \%(Simpson's) & 61.12 & 5.45 & 37.08 & 7.13 & 22.897 & $<0.001$ \\
\hline MAPSE Med (mm) & 11.96 & 2.23 & 7.34 & 2.51 & 11.465 & $<0.001$ \\
\hline MAPSE Lat. (mm) & 14.12 & 2.74 & 8.48 & 2.74 & 11.906 & $<0.001$ \\
\hline TD PSV Med. (cm/sec) & 9.31 & 1.85 & 6.08 & 1.77 & 10.217 & $<0.001$ \\
\hline TD PSV Lat. (cm/sec) & 9.93 & 2.06 & 6.6 & 1.7 & 9.862 & $<0.001$ \\
\hline
\end{tabular}

EF: Ejection Fraction; MAPSE: Mitral Annular Plane Systolic Excursion; TD: Tissue Doppler; PSV: Peak Systolic Velocity; Med.: Medial; Lat: Lateral.

Table 3: The sensitivity and specificity of the cut off values of MAPSE and Mitral annular TD PSV (Sm) at the Medial and Lateral Mitral annuli

\begin{tabular}{|c|c|c|c|c|c|c|}
\hline & Cut-off value & AUC & Sensitivity & Specificity & +PV & -PV \\
\hline MAPSE Med (mm) & $<=7$ & $93.20 \%$ & 73 & 100 & 100 & 78.8 \\
\hline MAPSE Lat (mm) & $<=10$ & $95 \%$ & 82 & 93 & 92.1 & 83.8 \\
\hline TD PSV Med (cm/sec) & $<=7$ & $93.20 \%$ & 91 & 85 & 85.8 & 90.4 \\
\hline TD PSV Lat (cm/sec) & $<=7$ & $90.50 \%$ & 72 & 93 & 91.1 & 76.9 \\
\hline
\end{tabular}

MAPSE: Mitral Annular Plane Systolic Excursion; TD: Tissue Doppler; PSV: Peak Systolic Velocity; Med.: Medial; Lat: Lateral.

Table 4: Combining Medial Mitral annular parameters (MAPSE and TD PSV) for predicting EF.

\begin{tabular}{|c|c|c|c|c|}
\hline & & \multicolumn{2}{|c|}{ Group } & \multirow{2}{*}{ Total } \\
\hline & & Group A & Group B & \\
\hline \multirow{2}{*}{ Combined medial annulus tests } & Prediction: Normal EF (>50\%) & 81 & 3 & 84 \\
\hline & Prediction: Impaired EF $(<50 \%)$ & 0 & 70 & 70 \\
\hline \multicolumn{2}{|c|}{ Total } & 81 & 73 & 154 \\
\hline
\end{tabular}

Sensitivity: $70 / 73=95.9 \%$, Specificity: $81 / 81=100 \%$ 
Table 5: Combining Lateral Mitral annular parameters (MAPSE and TD PSV) for predicting EF.

\begin{tabular}{|c|c|c|c|c|}
\hline & & \multicolumn{2}{|c|}{ Group } & \multirow{2}{*}{ Total } \\
\hline & & Group A & Group B & \\
\hline \multirow{2}{*}{ Combined lateral annulus tests } & Prediction: Normal EF (>50\%) & 88 & 11 & 99 \\
\hline & Prediction: Impaired EF $(<50)$ & 2 & 65 & 67 \\
\hline \multicolumn{2}{|c|}{ Total } & 90 & 76 & 166 \\
\hline
\end{tabular}

Sensitivity: 65/76= 85.5\%, Specificity: 88/90 = 97.7\%

\section{Discussion}

LV longitudinal shortening is a sensitive parameter reflecting cardiac systolic function. It contributes to about $60 \%$ of the LV stroke volume [9]. Because of their subendocardial location, the longitudinal fibers are more sensitive to affection by various diseases and pathologies [10]. Mitral annular plane systolic excursion (MAPSE) and TD PSV are surrogates for the LV longitudinal function [11-13] and there is a close correlation between them $[2,3]$. Both parameters are simple, easily measured and reproducible, even when performed by the beginner practitioners [12,14-16] and in patients with poor echogenicity when endocardial delineation is suboptimal [17-19] as well as in mechanically ventilated obese patients [20].

\section{MAPSE}

MAPSE has been suggested as a surrogate measurement for LVEF [14], where reduced MAPSE has been shown to correlate with LV systolic function in patients with myocardial infarction, heart failure, atrial fibrillation and critically ill patients with shock [21-23]. A recent study validated mitral annular plane systolic excursion (MAPSE)-derived formula to calculate the ejection fraction where $\mathrm{EF}=4.8 \times$ MAPSE $(\mathrm{mm})+5.8$ in adult males with left ventricular (LV) dysfunction [24]. MAPSE also provides important prognostic information in patients with cardiovascular disease $[21,23,25,26]$. Willenheimer et al. [11] showed that MAPSE has strong correlation to one-year mortality with $36 \%$ mortality in those with MAPSE $<6.4 \mathrm{~mm}$ as compared to freedom of mortality in those with MAPSE $>10 \mathrm{~mm}$ [11]. Similar results were found by Nikitin et al. [27]. In post-myocardial infarction patients with MAPSE $<8 \mathrm{~mm}$, the incidence of hospitalization and mortality was $43.8 \%$ [26]. Significant correlation was also found between MAPSE and serum BNP levels [12] and in a 10-year follow-up study, MAPSE was a strong risk predictor of long-term survival in heart failure [28].

The results of the present study showed the cut-off value for MAPSE at medial annulus $\leq 7 \mathrm{~mm}$ suggested an impaired systolic function with sensitivity of $73 \%$ and specificity of $100 \%$. While MAPSE at lateral annulus less $\leq 10 \mathrm{~mm}$ suggested an impaired systolic function with sensitivity of $82 \%$ and specificity of $93 \%$. These results correlate positively with the results of previous studies which showed that mean MAPSE of $\geq 10 \mathrm{~mm}$ was linked with normal EF ( $\geq 55 \%$ ) with a sensitivity of $90-92 \%$ and a specificity of $87 \%[29,30]$ and a value of MAPSE $<8 \mathrm{~mm}$ was associated with $\mathrm{EF}<50 \%$ with a specificity of $82 \%$ and a sensitivity of $98 \%$ [31] with values of lateral MAPSE generally higher than those of septal MAPSE [20,32]. In addition, a mean value for MAPSE of $\leq$
5 provided the best balanced sensitivity $(67.1 \%)$ and specificity $(76.5 \%)$ to predict $\mathrm{EF}<30 \%$ where a cut off value of $\leq 3.9$ could determine $\mathrm{EF}<30 \%$ in $100 \%$ of the patients [24].

\section{Mitral annular TD PSV}

Peak systolic velocity of mitral annular longitudinal movement $(\mathrm{Sm})$, provides an excellent non-invasive index of global $\mathrm{LV}$ systolic function with excellent correlation with established indexes [7]. It can also predict the LV wall motion abnormality during dobutamine stress echocardiography [33]. Moreover, the prognostic implication of peak systolic velocity of mitral annular longitudinal movement $(\mathrm{Sm})$ has been documented in patients with various cardiac diseases [27,34-37]. Sm is a strong predictor of cardiac mortality or rehospitalization for worsening of chronic heart failure in patients with LV dysfunction where $\mathrm{Sm}<2.8 \mathrm{~cm} /$ sec was associated with worse survival in patients with chronic heart failure and LV EFs $<45 \%[27,36]$.

The results of the present study showed the cut-off value of PSV (Sm) at the Medial and lateral annuli $\leq 7 \mathrm{~cm} / \mathrm{s}$ suggested impaired systolic function $(\mathrm{EF}<50)$ with sensitivity of $91 \%$ and specificity of $85 \%$ for the medial annulus and with sensitivity of $72 \%$ and specificity of $93 \%$ for the lateral annulus. These results correlate with Ruan and Nagueh who showed that Sm had strong correlation with LV EF and that $\mathrm{Sm}<7 \mathrm{~cm} / \mathrm{sec}$ was the most accurate parameter in identifying patients with LV EF $<45 \%$ (sensitivity, 93\%; specificity, 87\%) [38]. Vinereanu et al. [39] reported in 2002 a value of $8 \mathrm{~cm} / \mathrm{s}$ as a cut-off point for Sm for detection of LVEF $<50 \%$ with $80 \%$ sensitivity and $89 \%$ specificity at the medial mitral annulus and with $80 \%$ sensitivity and $92 \%$ specificity at the lateral Mitral annulus. However the study included only 51 patients who underwent radionuclide estimation of EF and without exclusion of patients with wall motion abnormalities [39]. The results of the present study does not correlate with Gulati et al. [16] who reported in 1996 average peak mitral annular velocity at both medial and lateral annuli is $>5.4 \mathrm{~cm} / \mathrm{s}$ as cut-off point for ejection fraction $>50 \%$ with $88 \%$ sensitivity and $97 \%$ specificity. The study included 55 patients, aged $56 \pm 15$ years, within 3 hours of radionuclide ventriculographic ejection fraction. Tissue Doppler studies were obtained from each of 6 mitral annular sites without exclusion of patients with wall motion abnormalities which could explain the difference from our results [16].

\section{Combined Mitral annular TD PSV and MAPSE}

To the best of our knowledge combing both parameters for prediction of EF was not reported before. In the present study 
when combining cut-off values of MAPSE and PSV for predicting impaired systolic function $(\mathrm{EF}<50)$ the sensitivity and specificity rose to $95.9 \%$ and $100 \%$ respectively for the medial Mitral annulus and $85.5 \%$ and $97.7 \%$ respectively for the lateral Mitral annulus suggesting that combining these 2 simple measurements can give more accurate, reliable and rapid information about the LV systolic function especially in patients with poor echogenicity.

\section{Conclusion}

In the present study, combining the measurement of both MAPSE and TD PSV increased their sensitivity and specificity for predicting the EF, suggesting that combining these 2 simple measurements can give more accurate, reliable and rapid information about the LV systolic function especially in patients with poor echogenicity. Further studies combining the measurement of both indices in different subsets of patients should be performed to confirm our results

\section{Conflict of Interest}

No financial interest or any conflict of interest exists.

\section{References}

1. Japp AG, Moir S, Mottram PM (2015) Echocardiographic Quantification of Left Ventricular Systolic Function. Heart Lung Circ 24(6): 532-535.

2. Lang RM, Bierig M, Devereux RB, Flachskampf FA, Foster E, et al (2005) Recommendations for chamber quantification: a re 7 port from the American Society of Echocardiogra $\urcorner$ phy's Guidelines and Standards Committee and the Chamber Quantification Writing Group, developed in conjunction with the European Association of Echocardiography, a branch of the European Society of Cardiology. J Am Soc Echocardiogr 18(12): 1440-1463.

3. Bellenger NG, Burgess MI, Ray SG, Lahiri A, Coats AJ, et al. (2000) Comparison of left ventricular ejection fraction and vo $ᄀ$ lumes in heart failure by echocardiography, radio $\urcorner$ nuclide ventriculography and cardiovascular ma $\mathrm{gnnetic} \mathrm{resonance;} \mathrm{are} \mathrm{they} \mathrm{interchangeable?}$ Eur Heart J 21(16): 1387-1396.

4. Brown J, Jenkins C, Marwick TH (2009) Use of myo cardial strain to assess global left ventricular func-tion: a comparison with cardiac magnetic resonan $\neg$ ce and 3-dimensional echocardiography. Am Heart J 157(1): 102.e1-5.

5. Jones CJ, Raposo L, Gibson DG (1990) Functional importance of the long axis dynamics of the human left ventricle. Br Heart J 63(4): 215-220.

6. Emilsson K, Wandt B (2000) The relation between mitral annulus motion and ejection fraction changes with age and heart size. Clin Physiol 20(1): 38-43.

7. Seo JS, Kim DH, Kim WJ, Song JM, Kang DH, et al. (2010) Peak systolic velocity of mitral annular longitudinal movement measured by pulsed tissue Doppler imaging as an index of global left ventricular contractility. Am J Physiol Heart Circ Physiol 298(5): H1608-1615.

8. Lang RM, Badano LP, Mor-Avi V, Afilalo J, Armstrong A, et al (2015) Recommendations for cardiac chamber quantification by echocardiography in adults: an update from the American Society of Echocardiography and the European Association of Cardiovascular Imaging. J Am Soc Echocardiogr 28(1): 1-39.e14.
9. Carlsson M, Ugander M, Mosén H, Buhre T, Arheden H. (2007) Atrioventricular plane displacement is the major contributor to left ventricular pumping in healthy adults, athletes, and patients with dilated cardiomyopathy. Am J Physiol Heart Circ Physiol 292(3): H1452-1459.

10. Henein MY, Gibson DG (1999) Long axis function in disease. Heart 81(3): 229-231.

11. Willenheimer R, Cline C, Erhardt L, Israelsson B (1997) Left ventricular atrioventricular plane displacement: an echocardiographic technique for rapid assessment of prognosis in heart failure. Heart 78(3): 230-236.

12. Elnoamany MF, Abdelhameed AK (2006) Mitral annular motion as a surrogate for left ventricular function: correlation with brain natriuretic peptide levels. Eur J Echocardiogr 7(3): 187-198.

13. Mogelvang R, Sogaard P, Pedersen SA, Olsen NT, Schnohr P, et al. (2009) Tissue Doppler echocardiography in persons with hypertension, diabetes, or ischaemic heart disease: the Copenhagen City Heart Study. Eur Heart J 30(6): 731-739.

14. Willenheimer R (1998) Assessment of left ventricular dysfunction and remodeling by determination of atrioventricular plane displacement and simplified echocardiography. Scand Cardiovasc J Suppl 48: 1-31.

15. Matos J, Kronzon I, Panagopoulos G, Perk G (2012) Mitral annular plane systolic excursion as a surrogate for left ventricular ejection fraction. J Am Soc Echocardiogr 25(9): 969-974.

16. Gulati VK, Katz WE, Follansbee WP, Gorcsan J 3rd (1996) Mitral annular descent velocity by tissue Doppler echocardiography as an index of global left ventricular function. Am J Cardiol 77(11): 979-984.

17. Hu K, Liu D, Herrmann S, Niemann M, Gaudron PD, et al. (2013) Clinical implication of mitral annular plane systolic excursion for patients with cardiovascular disease. Eur Heart J Cardiovasc Imaging 14(3): 205-212.

18. Yuda S, Inaba Y, Fujii S, Kokubu N, Yoshioka T, et al. (2006) Assessment of Left Ventricular Ejection Fraction Using LongAxis Systolic Function Is Independent of Image Quality: A Study of Tissue Doppler Imaging and M-Mode Echocardiography. Echocardiography 23(10): 846-852.

19. Shimizu Y, Uematsu M, Shimizu H, Nakamura K, Yamagishi M, et al. (1998) Peak negative myocardial velocity gradient in early diastole as a noninvasive indicator of left ventricular diastolic function: comparison with transmitral flow velocity indices. J Am Coll Cardiol 32(5): 1418-1425.

20. Zidan DH, Helmy TA (2016) Usefulness of mitral annular plane systolic excursion in assessment of left ventricular systolic function in mechanically ventilated obese patients. J Crit Care 34: 74-76.

21. Alam M, Höglund C, Thorstrand C, Philip A. (1990) Atrioventricular plane displacement in severe congestive heart failure following dilated cardiomyopathy or myocardial infarction. J Intern Med 228(6): 569-575.

22. Emilsson K, Wandt B (2000) The relation between ejection fraction and mitral annulus motion before and after direct-current electrical cardioversion. Clin Physiol 20(3): 218-224.

23. Bergenzaun L, Ohlin H, Gudmundsson P, Willenheimer R, Chew MS (2013) Mitral annular plane systolic excursion (MAPSE) in shock: a valuable echocardiographic parameter in intensive care patients. Cardiovasc Ultrasound 11:16. 
24. Adel W, Roushdy AM, Nabil M (2016) Mitral Annular Plane Systolic Excursion-Derived Ejection Fraction: A Simple and Valid Tool in Adult Males With Left Ventricular Systolic Dysfunction. Echocardiography 33(2): 179-184

25. Höglund C, Alam M, Thorstrand C. (1988) Atrioventricular valve plane displacement in healthy persons. An echocardiographic study. Acta Med Scand 224(6): 557-562

26. Brand B, Rydberg E, Ericsson G, Gudmundsson P, Willenheimer R (2002) Prognostication and risk stratification by assessment of left atrioventricular plane displacement in patients with myocardial infarction. Int J Cardiol 83(1): 35-41.

27. Nikitin NP, Loh PH, Silva R, Ghosh J, Khaleva OY, et al. (2006) Prognostic value of systolic mitral annular velocity measured with Doppler tissue imaging in patients with chronic heart failure caused by left ventricular systolic dysfunction. Heart 92(6): 775779

28. Sveälv BG, Olofsson EL, Andersson B (2008) Ventricular long-axis function is of major importance for long-term survival in patients with heart failure. Heart 94(3): 284-289.

29. Alam M, Höglund C, Thorstrand C (1992) Longitudinal systolic shortening of the leftventricle: an echocardiographic study in subjects with and without preserved global function. Clin Physio 12(4): 443-452.

30. Silva JA, Khuri B, Barbee W, Fontenot D, Cheirif J (1996) Systolic excursion of the mitral annulus to assess septal function in paradoxic septal motion. Am Heart J 131(1): 138-145.

31. Simonson JS, Schiller NB (1989) Descent of the base of the left ventricle: an Echocardiographic index of left ventricular function. J Am Soc Echocardiogr 2(1): 25-35.
32. Carlhäll C, Wigström L, Heiberg E, Karlsson M, Bolger AF, et al. (2004) Contribution of mitral annular excursion and shape dynamics to total left ventricular volume change. Am J Physiol Heart Circ Physiol 287(4): H1836-1841.

33. Sharif D, Sharif-Rasslan A, Shahla C (2011) Mitral Annular Systolic Velocities Predict Left Ventricular Wall Motion Abnormality During Dobutamine Stress Echocardiography. Cardiol Res 2(1): 16-26.

34. Agricola E, Galderisi M, Oppizzi M, Schinkel AF, Maisano F, et al. (2004) Pulsed tissue Doppler imaging detects early myocardial dysfunction in asymptomatic patients with severe mitral regurgitation. Heart 90(4): 406-410.

35. Marwick TH, Case C, Leano R, Short L, Baglin T, et al. (2004) Use of tissue Doppler imaging to facilitate the prediction of events in patients with abnormal left ventricular function by dobutamine echocardiography. Am J Cardiol 93(2): 142-146.

36. Wang M, Yip GW, Wang AY, Zhang Y, Ho PY, et al. (2003) Peak early diastolic mitral annulus velocity by tissue Doppler imaging adds independent and incremental prognostic value. J Am Coll Cardiol 41(5): 820-826.

37. Wang M, Yip GW, Wang AY, Zhang Y, Ho PY, et al. (2005) Tissue Doppler imaging provides incremental prognostic value in patients with systemic hypertension and left ventricular hypertrophy. J Hypertens 23(1): 183-191.

38. Ruan Q Nagueh SF (2006) Usefulness of isovolumic and systolic ejection signals by tissue Doppler for the assessment of left ventricular systolic function in ischemic or idiopathic dilated cardiomyopathy. Am J Cardiol 97(6): 872-875.

39. Vinereanu D, Khokhar A, Tweddel AC, Cinteza M, Fraser AG (2002) Estimation of global left ventricular function from the velocity of longitudinal shortening. Echocardiography 19(3): 177-185. 